Recepción: 20 / 04 / 2017

Aceptación: 20 / 05 / 2017

Publicación: 15 / 07 / 2017

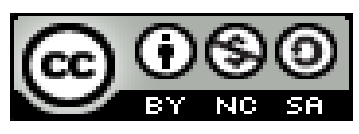

Ciencias Económicas y empresariales Artículo Científico

\title{
Valoración de flujos futuros mediante el uso eficiente del costo promedio ponderado de capital (WACC)
}

\author{
Valuation of future flows through the efficient use of the weighted average cost of \\ capital (WACC)
}

\section{Avaliação do futuro flui através do uso eficiente do custo médio ponderado de capital (WACC)}

\author{
Luis M. Bejar-León ${ }^{\mathrm{I}}$ \\ luis.bejarl@ug.edu.ec \\ Elaine R. Jijon-Gordillo ${ }^{\text {II }}$ \\ elainejijon@hotmail.com \\ Vladimir J. Soria-Freire ${ }^{\text {III }}$ \\ vladimir.soriaf@ug.edu.ec
}

Correspondencia: luis.bejarl@ug.edu.ec

\footnotetext{
I. Magister en Negocios Internacionales y Gestión de Comercio Exterior; Diploma Superior en Pedagogía Universitaria; Diploma Superior en Economía Internacional; Ingeniero Comercial; Universidad de Guayaquil, Guayaquil, Ecuador. II. Magister en Administración de Negocios; Magister en Administración de Empresas; Economista; Universidad de Guayaquil, Guayaquil, Ecuador.

III. Maestro en Economía con Mención en Economía Ecológica; Diploma Superior en Pedagogía Universitaria; Abogado de los Tribunales y Juzgados de la República del Ecuador; Universidad de Guayaquil, Guayaquil, Ecuador.
} 


\section{Resumen}

Las empresas, generalmente, obtienen financiamiento de sus activos mediante deudas a largo plazo. En el caso de que se dé esta premisa, su costo de capital debe reflejar el costo promedio de las diversas fuentes de financiamiento que se hayan usado. Las partidas que aparecen en el lado derecho del Balance General (los diversos tipos de deudas y capital contable) son los componentes de financiamiento de una empresa. Cualquier incremento en los activos totales debe ser financiado mediante un aumento en uno o más de los componentes de financiamientos. El objetivo del presente artículo es demostrar que, con el correcto uso del WACC (Costo Promedio Ponderado de Capital), se obtendrá una mejor y confiable valoración de los flujos proyectados. Este instrumento financiero permite encontrar el costo promedio de las diversas fuentes de financiamiento, mediante el uso de ponderaciones que representan la participación de cada fuente en relación al financiamiento total. Este trabajo estará basado bajo el método analítico - deductivo, debido a la importancia que tiene cada componente a tratarse y a su vez erradicar los posibles errores en el uso del WACC, ya que de esta dependerá la validación de los flujos y en efecto de la empresa. El uso eficiente del Costo Promedio Ponderado de Capital (WACC) permite a las empresas valorar los flujos de una forma más real y confiable.

Palabras claves: Costo de capital; WACC; Fuentes de financiamiento; balance general. 


\begin{abstract}
Companies generally obtain financing of their assets through long-term debt. In the event that this premise is given, its cost of capital must reflect the average cost of the various sources of financing that have been used. The items that appear on the right side of the Balance Sheet (the various types of debts and stockholders' equity) are the financing components of a company. Any increase in total assets must be financed by an increase in one or more of the financing components. The objective of the present article is to demonstrate that, with the correct use of the WACC (Average Weighted Cost of Capital), a better and reliable valuation of the projected flows will be obtained. This financial instrument makes it possible to find the average cost of the various sources of financing, using weights that represent the participation of each source in relation to the total financing. This work will be based on the analytic - deductive method, due to the importance of each component to be addressed and in turn to eradicate possible errors in the use of the WACC, since this will depend on the validation of the flows and in effect of the company. The efficient use of the Weighted Average Cost of Capital (WACC) allows companies to value flows in a more real and reliable way.
\end{abstract}

Key words: Cost of capital; WACC; sources of financing; balance sheet. 


\section{Resumo}

As empresas geralmente obtêm o financiamento de seus ativos através de dívida de longo prazo. No caso de esta premissa ser dada, o seu custo de capital deve refletir o custo médio das várias fontes de financiamento que foram utilizadas. Os itens que aparecem no lado direito do balanço (os vários tipos de dívidas e patrimônio líquido) são os componentes de financiamento de uma empresa. Qualquer aumento nos ativos totais deve ser financiado por um aumento em um ou mais dos componentes de financiamento. O objetivo do presente artigo é demonstrar que, com o uso correto do WACC (custo médio ponderado de capital), será obtida uma avaliação melhor e confiável dos fluxos projetados. Este instrumento financeiro permite encontrar o custo médio das diversas fontes de financiamento, utilizando pesos que representam a participação de cada fonte em relação ao financiamento total. Este trabalho basear-se-á no método analítico-dedutivo, devido à importância de cada componente a ser abordado e, por sua vez, para erradicar possíveis erros no uso do WACC, uma vez que isso dependerá da validação dos fluxos e de efeito de a empresa . O uso eficiente do custo médio ponderado de capital (WACC) permite que as empresas avaliem os fluxos de forma mais real e confiável.

Palavras-chave: Custo de capital; WACC; fontes de financiamento; balanço patrimonial. 


\section{Introducción.}

El principal objetivo de una empresa, ya consolidada, es mantenerse por la senda del crecimiento financiero, es decir incrementar cada vez más sus márgenes de rentabilidad, lo que en su efecto crearía mayor valor a sus acciones. Para la verificación de aquello se necesita de herramientas financieras que permitan obtener una correcta y confiable valoración de la empresa. Una de las herramientas más importante es la valoración de los flujos que posee la empresa, pero esta deberá contar con la presencia de una tasa de descuento eficiente para que así los resultados sean lo más reales posibles. Pero, ¿los empresarios y/o emprendedores identifican a la tasa de descuento más completa y eficiente para la valoración de sus flujos?

Uno de los problemas más difíciles en la valoración de proyectos es la necesidad de conocer y escoger la tasa de descuento apropiada que hará que sus flujos viabilicen la factibilidad financiera y económica del proyecto. Este es un problema al que se enfrentan todos los analistas que desean exponer sus resultados como confiables y viables. Algunos inversionistas en su afán de valorar mejor sus inversiones, utilizan métodos de valoración que se descuentan a una tasa de rentabilidad esperada, pero ninguna que les asegure su cercanía con ser la apropiada para esa actividad en referencia.

Generalmente cuando se trata de exponer la relevancia de un proyecto, los emprendedores se encuentran con la difícil tarea de explicar el motivo por la cual se escogió la tasa de descuento. A partir de la aplicación de esta concepción, será más fácil la exposición de cualquier proyecto de inversión planteando que el financiarse de distintas fuentes favorece el ponderar el costo de capital. Entre más fuentes de financiamiento externo e interno se utilice, la medida de costos de capital será mejor para la valoración de los flujos de fondos. 
Para escoger una tasa de interés o descuento adecuada para su proyecto, es importante determinar los recursos financieros y económicos que necesitaran sus flujos, para luego determinar el costo de capital promedio que descontado hace factible su inversión. En el presente trabajo, se demostrará que haciendo uso de una tasa promedio ponderada de deudas y capital permitirá asegurar la confianza en la valoración de los flujos futuros, es decir que la correcta selección de esa tasa fortalecerá el marco de valoración interna de fondos proyectados. En otras palabras, el objetivo principal es demostrar y comprobar, mediante un estudio de caso, que el uso del WACC asegura la obtención de flujos de fondos futuros más reales y factibles.

Las variables que estarán presente en la investigación, son exclusivamente financieras por lo que a continuación se presenta las definiciones de las mismas:

Costo de Capital: Es la tasa mínima de rentabilidad que deben proporcionar las inversiones de las entidades para que por lo menos mantenga el mismo nivel el valor de las acciones dentro del mercado de capitales.

$\underline{W A C C}$ : Es la tasa de descuento que suele emplearse para descontar los flujos de fondos operativos para valorar una empresa utilizando el descuento de flujos de efectivo, en el "enterprise approach".

Fuentes de Financiamiento: Es el conjunto de capital interno y externo a la organización utilizado para financiar los activos, capital de trabajo, entre otros. La disyuntiva estará en financiarse interna o externamente, capital contable o deuda con terceros.

Balance General: Es un estado financiero compuesto por un documento que detalla los activos, pasivos y patrimonio con los que cuenta la empresa en un periodo determinado. 


\section{Metodología.}

El realizar una investigación, ya sea de ámbito económico, social, empresarial u otro, requiere de la elección de un modelo o diseño para la consecución de información eficiente para el cumplimiento de objetivos que se proponga el investigador. En el caso de esta investigación, es una indagación netamente cuantitativa, es decir objetiva con bases numéricas y a la vez el uso del estudio de casos para su mejor entendimiento, lo que permitirá al lector un mayor manejo de las concepciones que se brindará en el texto.

Además, la investigación se llevará a cabo bajo el método analítico - deductivo, ya que se procederá a descomponer las partes de los flujos y asimismo las formas de cálculo de las tasas de descuentos correspondientes. Por otro lado, también será de tipo descriptivo ya que se analizará las diferentes tasas de descuentos que se utilizan para la valoración de flujos en los proyectos de inversión.

En suma, el desarrollo de la investigación se lo hará mediante el enfoque cuantitativo usando variables netamente financieras por lo que no será necesario operacionalizar las variables para el cumplimiento de los objetivos de la investigación. Además, la investigación experimental ${ }^{1}$ se hará presente en el desarrollo del texto, ya que el estudio de caso será pertinente para un mayor entendimiento por parte del lector. Además, se utilizarán libros, artículos científicos, entre otros

\footnotetext{
${ }^{1}$ Véase más en: Hernández Sampieri, R., Fernández Collado, C., \& Baptista Lucio, P. (2010). Metodología de la investigación. México: McGraw-Hill Interoamericana.
} 
textos para el desarrollo de la investigación, por lo que también se nota la presencia de una investigación de tipo documental o bibliográfica.

\section{Discusión.}

\section{Discriminación de Tasa de Descuento para cada Flujo de Fondos}

Uno de los dilemas comunes cuando valoramos un flujo de fondos, es elegir la tasa de descuento adecuada para calcular el valor actual de los flujos netos después de impuestos. Previamente a escoger una tasa de descuento, definamos los personajes para quien se realiza la valoración:

- La empresa,

- El aportante de recursos ajenos, y

- El aportante de recursos propios.

Conocer el interés de esta evaluación es necesario ya que cada uno tiene diferentes medidas de rentabilidad en la inversión.

Los estados financieros (Balance General, Estado de Resultados y Estado de Flujo de Efectivo) proporcionarán la información para la elaboración del flujo. Sin embargo, se debe realizar ciertos ajustes a la utilidad neta debido a que los Estados Financieros no proveen información exacta de los movimientos del efectivo ${ }^{2}$; y como complemento para conocer las fuentes y usos del dinero, se debe preparar el Estado de Flujos de Efectivo $^{3}$.

Flujo de Fondos Libre o Free Cash Flow (FCF)

\footnotetext{
${ }^{2}$ Si la empresa vende y paga todo en efectivo, entonces los estados financieros mostrarían exactamente los movimientos del efectivo.

${ }^{3}$ El Estado de Flujos de Efectivo, explica la generación o uso de efectivo de acuerdo a la actividad de la empresa: Operación, Inversión y Financiamiento
} 
El Flujo de fondos libre (FCF) representa el flujo de fondos que genera la empresa, independientemente de cómo está financiada. Los flujos a evaluarse deberán estar libres de pagos de intereses y amortizaciones de deudas

Por tales motivos, el Flujo de Fondos utilizado es denominado Flujo Económico Puro o Flujo de Fondos Libre (de deudas e intereses) ${ }^{4}$. Este flujo se obtiene, partiendo de la utilidad neta ${ }^{5}$ y realizando los respectivos ajustes:

Tabla 1. Obtención del Flujo de Fondos Libre

\begin{tabular}{|c|c|c|}
\hline Free Cash Flow (FCF) & & \\
\hline Ingresos o Ventas & & \\
\hline - Costo de ventas/producción & & \\
\hline Utilidad Bruta & & \\
\hline - $\quad$ Gastos Administrativos & & \\
\hline - $\quad$ Gastos Ventas & & \\
\hline Utilidad antes de Intereses, & & \\
\hline Impuestos, Depreciación y & & \\
\hline Amortización (EBITDA) & & \\
\hline - Depreciación & & \\
\hline - $\quad$ Amortización & & \\
\hline Utilidad antes de Intereses e & & Utilidad antes de Intereses e \\
\hline Impuestos (EBIT) & & Impuestos (EBIT) \\
\hline - $\quad$ Gastos Financieros & & \\
\hline $\begin{array}{l}\text { Utilidad antes de Impuestos } \\
\text { (EBT) }\end{array}$ & & \\
\hline - $\quad$ Impuestos sobre la Renta & & \\
\hline Utilidad Neta (NI) & & \\
\hline$+\quad$ Depreciación & + & Depreciación \\
\hline$+\quad$ Amortización intangibles & + & Amortización intangibles \\
\hline$+\quad$ Gastos Financieros & - & Impuestos \\
\hline - $\quad$ Escudo Fiscal (GF*T) & & Escudo Fiscal (GF*T) \\
\hline - $\quad$ Variaciones de Capital de trabajo & - & Variaciones de Capital de trabajo \\
\hline
\end{tabular}

\footnotetext{
${ }^{4}$ No se consideran los intereses porque el valor tiempo del dinero proveniente de los recursos ajenos, debería estar considerado en la tasa de descuento del FCF. No se toman en cuenta las amortizaciones de la deuda porque, si la inversión inicial está compuesta en parte por recursos ajenos, al incluir las amortizaciones estaríamos castigando la inversión inicial dos veces.

${ }^{5}$ Se puede partir de la utilidad neta como del resultado operativo (EBIT). Si se parte del primero se suman los gastos financieros; si se parte del segundo se restan los impuestos.
} 
Valoración de flujos futuros mediante el uso eficiente del costo promedio ponderado de capital (WACC)

+/- Venta/Compra de Activos Fijos Flujo de Fondos Libre (FCF)
+/- Venta/Compra de Activos Fijos Flujo de Fondos Libre (FCF)

Tasa de Descuento apropiada para el FCF

La tasa de descuento que debe aplicarse para el FCF es el Costo Promedio Ponderado de Capital y se obtiene de la siguiente manera:

- El costo de la deuda, puesto que los intereses no están incluidos en el FCF

- La proporción de la deuda como parte del financiamiento de la inversión (peso de la deuda)

- El escudo o crédito fiscal que causan los intereses (tampoco están incluidos en el FCF)

- La rentabilidad que desea obtener el inversionista

- La Proporción de los recursos propios como parte del financiamiento de la inversión (peso de patrimonio)

El costo promedio ponderado de capital, $\mathrm{WACC}^{6}$, se calcula con la siguiente fórmula:

$$
W A C C=K_{d} \frac{\nu}{D+E}(1-T)+K_{e} \frac{E}{D+E}
$$

Dónde:

\footnotetext{
${ }^{6}$ WACC, Weighted Average Cost of Capital. Véase Miles, James y John R. Ezzel (1980), “The Weighted Average Cost of Capital, Perfect Capital Markets and Project Life: A Clarification”, en Journal of Financial and Quantitative Analysis, Septiembre: 719-730.
} 
Tabla 2. Nomenclatura de la Ecuación 1

\begin{tabular}{lcl}
\hline \multicolumn{2}{l}{ Significado por partes de la Ecuación 1} & \\
\hline $\mathrm{D} /(\mathrm{D}+\mathrm{E})$ & $K_{d}$ & $\begin{array}{l}\text { Costo de la deuda } \\
\text { Proporción de la deuda con respecto a la } \\
\text { inversión }\end{array}$ \\
& & $\begin{array}{l}\text { Escudo o Crédito Fiscal } \\
\text { Rentabilidad esperada por el inversionista } \\
\text { (costo del Patrimonio o Equity) } \\
\end{array}$ \\
& $K_{e}$ & $\begin{array}{l}\text { Proporción de los recursos propios con } \\
\text { respecto a la inversión }\end{array}$ \\
$\mathrm{E} /(\mathrm{D}+\mathrm{E})$ & &
\end{tabular}

Flujo de Fondos de Capital o Capital Cash Flow (CCF)

El Flujo de fondos de capital (CCF) se define como el flujo total para los inversionistas. Es la suma de los flujos de fondos que éstos perciben:

- Dividendos para los accionistas, y

- Cambios en el nivel de deuda e intereses para los prestamistas.

También puede ser obtenido a partir del FCF: puesto que el FCF no considera el Escudo o Crédito Fiscal, sumamos éste para llegar al CCF:

Tabla 3. Obtención del Capital Cash Flow

Flujo de Fondos Libre (FCF)

+ Escudo Fiscal $\left(\mathrm{GF}^{*} \mathrm{~T}\right)$

Flujo de Fondos de Capital (CCF)

Tasa de descuento apropiada para el CCF

La tasa de descuento a utilizarse para el CCF deberá tener en consideración todos los puntos señalados anteriormente para el descuento del FCF, con la excepción del Escudo Fiscal, puesto que 
éste ya está siendo sumado al FCF para obtener el CCF. Por tanto, la tasa de descuento a aplicarse deberá ser un WACC antes de impuestos ${ }^{7}$ :

$$
\text { WACG }_{B T}=K_{d} \frac{D}{D+E}+K_{e} \frac{E}{D+E}
$$

Dónde:

Tabla 4. Nomenclatura de la Ecuación 2

\begin{tabular}{lcl}
\hline Significado por partes de la Ecuación 2 & \\
\hline $\mathrm{D} /(\mathrm{D}+\mathrm{E})$ & $K_{d}$ & $\begin{array}{l}\text { Costo de la deuda } \\
\text { Proporción de la deuda con respecto a la } \\
\text { inversión }\end{array}$ \\
& $K_{e}$ & $\begin{array}{l}\text { Rentabilidad esperada por el inversionista } \\
\text { (costo del Patrimonio o Equity) } \\
\text { Proporción de los recursos propios con } \\
\text { respecto a la inversión }\end{array}$ \\
\hline
\end{tabular}

Flujo de fondos para el inversionista/accionista o Equity Cash Flow (ECF)

En el Flujo de fondos para el inversionista (ECF) se debe considerar un flujo de fondos residual, es decir el resultado del Flujo de fondos de capital (CCF) restando los intereses y las amortizaciones de deuda:

Los accionistas o inversionistas institucionales son los mayores interesados en este flujo con el objetivo de conocer la rentabilidad de financiar a la empresa en proyectos nuevos. Es necesaria la comparación de la inversión versus los flujos de fondos que percibirá a través del tiempo.

\footnotetext{
${ }^{7}$ WACC $_{B T}$, WACC before taxes.
} 
Tabla 5. Obtención del ECF

\begin{tabular}{llll}
\hline Flujo & Fondos de Capital \\
(CCF)
\end{tabular}

- Amortización Deuda

- Gastos Financieros

+ Recursos Externos ${ }^{8}$

\section{Flujo del Inversionista (ECF)}

\section{Tasa de Descuento apropiada para el ECF}

Se debe utilizar la rentabilidad que el inversionista espera obtener por su inversión. Para que se justifique la decisión de invertir en la empresa el accionista exige un rendimiento mayor a la rentabilidad que pueda obtener en títulos financieros libres de riesgos.

Invertir en un proyecto o empresa conlleva en incurrir a ciertos riesgos, y en consecuencia la rentabilidad del inversionista debería ser igual a la tasa libre de riesgo más una prima por riesgo; por lo tanto, podemos definir la rentabilidad del accionista a través de dos métodos:

- Modelo de equilibrio de activos financieros o también conocido como modelo de fijación de precios para activos de capital CAPM ${ }^{9}$ :

$K_{e}=R_{f}+\beta_{e}\left(R_{m}-R_{f}\right)$

\section{Donde:}

\footnotetext{
${ }^{8}$ Para encontrar la TIR del proyecto la inversión total deberá estar con signo negativo, al sumarle los recursos externos, se encontrará la diferencia que corresponderá a la inversión propia.

${ }^{9}$ CAPM, Capital Asset Pricing Model. Véase Sharpe, William (1964): "Capital Asset Prices: A Theory of Market Equilibrium Ander Condition of Risk", en Journal of Finance, septiembre: 277-293.
} 
Tabla 6. Nomenclatura de la ecuación 3

Significado por partes de la Ecuación 3

$\begin{array}{cl}R_{f} & \text { Rendimiento o tasa libre de riesgo (Risk } \\ & \text { Free) } \\ \beta_{e} & \text { Proporción del riesgo, coeficiente beta } \\ R_{m} & \text { Riesgo del mercado, índice de mercado } \\ \left(R_{m}-R_{f}\right) & \text { Prima de Riesgo del mercado }\end{array}$

- Modelo de capitalización de dividendos por Gordon y Shapiro:

$K_{e}=\left[D I V_{1} / P_{0}\right]+g$

$K_{e}=\left[D I V_{0}(1+g) / P_{0}\right]+g$

Tabla 7. Nomenclatura de la ecuación 5

\begin{tabular}{cl}
\hline Significado por partes de la Ecuación 5 & \\
\hline $\operatorname{Div}_{1}$ & Valor de dividendo a recibir en el \\
& siguiente año [Div1 = Div0 (1+g)] \\
$P_{0}$ & Precio actual de la acción \\
$g$ & Tasa de crecimiento constante y \\
& sostenible de los dividendos \\
\hline
\end{tabular}

Costo Promedio Ponderado de Capital/Weighted Average Cost of Capital (WACC) 
Costo de deudas por financiamiento

El Costo de la Deuda resulta de la tasa de interés sobre la deuda (tasa activa) menos los ahorros fiscales que resultan, puesto que el interés es un gasto deducible. La fórmula para obtener el costo de la deuda es:

$$
K_{d}(1-T)
$$

Dónde:

Tabla 8. Nomenclatura del costo de la deuda

\begin{tabular}{cl}
\hline Nomenclatura de la Ecuación 2 & \\
\hline$K_{d}$ & $\begin{array}{l}\text { Tasa de interés sobre la deuda (tasa } \\
\text { activa) }\end{array}$ \\
$(1-T)$ & Impuestos (Taxes) \\
\hline
\end{tabular}

Ejemplos:

Si se solicitara un préstamo por $\$ 500,000.00$ a una tasa del $16 \%$, considerando el $25 \%$ de impuestos y por el plazo de 1 año, entonces el costo de la deuda después de impuestos sería:

Tabla 9. Resolución de ejemplo sobre costo de la deuda

$\begin{array}{ll}K_{d}(1-T) & 16 \%(1-0.25) \\ K_{d}(1-T) & 16 \%(0.75) \\ K_{\boldsymbol{d}}(\mathbf{1}-\boldsymbol{T}) & \mathbf{1 2 \%}\end{array}$

Pol. Con. (Edición núm. 9) Vol. 2, No 7, julio, 2017, pp.156-189, ISSN: 2550 - 682X 
Esto quiere decir que el costo de la deuda sería del $12 \%$, puesto que el estado paga el $4 \%$ porque el interés es un gasto deducible. El gasto deducible de los impuestos, es visible en los resultados de la empresa.

Tabla 10. Estructura del Estado de Resultado con/sin deuda

\section{Estado de Resultados}

\begin{tabular}{lll}
\hline & Con Deuda & Sin Deuda \\
Ventas & 800.000 .00 & 800.000 .00 \\
Costos de Ventas & 300.000 .00 & 300.000 .00 \\
Utilidad Bruta & $\mathbf{5 0 0 . 0 0 0 . 0 0}$ & $\mathbf{5 0 0 . 0 0 0 . 0 0}$ \\
Gastos Generales & 150.000 .00 & 150.000 .00 \\
Gastos Financieros & $\mathbf{8 0 . 0 0 0 . 0 0}$ & $\mathbf{0 . 0 0}$ \\
Utilidad antes & 270.000 .00 & 350.000 .00 \\
Impuestos & & $\mathbf{8 7 . 5 0 0 . 0 0}$ \\
Impuestos (25\%) & $\mathbf{6 7 . 5 0 0 . 0 0}$ & $\mathbf{2 6 2 . 5 0 0 . 0 0}$ \\
Utilidad neta & $\mathbf{2 0 2 . 5 0 0 . 0 0}$ & \\
Utilidad neta (sin deuda) & 262.500 .00 & \\
Utilidad neta (con deuda) & 202.500 .00 & \\
Escudo Fiscal & $\mathbf{6 0 . 0 0 0 . 0 0}$ & \\
Ahorro Fiscal & Gtos. Financieros $(1-\mathrm{T})$ & \\
& $80.000 .00 \times(1-0.25)$ & \\
Financiamiento & $\mathbf{6 0 . 0 0 0 . 0 0}$ & \\
Ahorro Fiscal & 80.000 .00 & \\
Desembolso & 60.000 .00 & \\
500.000.00 x 16\% & $\mathbf{2 0 . 0 0 0 . 0 0}$ & \\
500.000.00 x 4\% & 80.000 .00 & \\
500.000.00 x 12\% & 20.000 .00 & \\
\hline & $\mathbf{6 0 . 0 0 0 . 0 0}$ & \\
\hline & & \\
\hline & & \\
\hline
\end{tabular}


La razón para usar el costo de deudas después de impuestos es la siguiente: el valor de las acciones de la empresa, el cual se desea maximizar, depende de los flujos de efectivo después de impuestos. Cada vez que el interés sea un gasto deducible, produce ahorros en impuestos que reducen el costo componente de la deuda, haciendo que el costo de las deudas después de impuestos sea inferior al costo antes de impuestos.

Obsérvese que el costo de las deudas es igual a la tasa activa sobre las deudas nuevas, no al interés de las deudas contraídas y pendientes de pago. El principal interés en el costo de capital es para decisiones de presupuesto de capital. La tasa a la cual la empresa haya solicitado préstamos en el pasado, representa un costo hundido para la empresa y es irrelevante para propósitos de costo de capital. La tasa realmente importante es la tasa a la que se pueda solicitar un préstamo en el futuro.

\section{Costo del Capital Contable Interno (Patrimonio).}

El capital contable interno (patrimonio) está compuesto por:

- Capital Social, dividido en acciones

- Utilidades del ejercicio en curso

- Utilidades Retenidas

Así como se puede financiar proyectos mediante pasivos a largo plazo, también se puede financiar mediante el patrimonio y la forma como se obtiene ingresos en el patrimonio, es a través de las utilidades retenidas. Por tanto, el costo de las utilidades retenidas es la tasa de rendimiento que requieren los accionistas sobre el capital contable que obtiene la empresa al retener utilidades.

La razón por la cual se debe asignar un costo de capital a las utilidades retenidas se relaciona con el Principio del Costo de Oportunidad. Las utilidades pertenecen exclusivamente a los 
accionistas; estas utilidades sirven para compensar a los accionistas por el uso de su capital. La directiva puede decidir si pagar las utilidades a los accionistas en forma de dividendos o puede retenerlas y reinvertirlas dentro del negocio.

Al retener las utilidades, existirá un costo de oportunidad, puesto que los accionistas podrían reinvertir ese dinero en bonos, bienes raíces o cualquier otra clase de activos. Por tanto, la empresa debería ganar sobre sus utilidades retenidas por lo menos, tanto como lo que sus accionistas pudieran ganar sobre inversiones alternativas de riesgo comparable.

Existen tres métodos para calcular el costo del patrimonio:

- Método de Mercado.- Puesto que las utilidades retenidas son de los accionistas, quienes desean la maximización del precio de sus acciones, la tasa de rendimiento esperada sobre las utilidades retenidas debe ser igual a la tasa de rendimiento requerida sobre las acciones. Tal como se estudió, para calcular el rendimiento requerido de las acciones y por ende de las utilidades retenidas, se usa la ecuación del CAPM:

$$
K_{e}=T L R+\beta\left(R_{m}-T L R\right)
$$

- Dividendos más Tasa de Crecimiento (Flujo Descontado). - Tanto el precio como el rendimiento esperado sobre una acción, dependen en última instancia, de los dividendos esperados sobre la acción: 


$$
P=\frac{D}{\left(1+K_{e}\right)^{n}}
$$

En este caso, el precio de la acción (P) es el valor descontado del dividendo D que se espera que se pague al final del año $\mathrm{n}$, a una tasa de descuento equivalente al rendimiento esperado o costo de capital $\mathrm{K}_{\mathrm{e}}$. Si se esperan que los dividendos crezcan a una tasa constante, entonces el precio de la acción se definiría como:

$$
\mathrm{P}=\frac{\mathrm{D}}{\mathrm{K}_{\mathrm{e}}-\mathrm{g}}
$$

Despejando Ke, se obtiene la tasa esperada de rendimiento:

$$
\mathrm{K}_{\mathrm{e}}=\frac{\mathrm{D}}{\mathrm{P}}+\mathrm{g}
$$

De tal forma, los inversionistas esperan recibir un rendimiento por dividendos $(\mathrm{D} / \mathrm{P})$ más una ganancia en capital (g), para obtener un rendimiento total esperado $\mathrm{K}_{\mathrm{e}}$. La situación típica de toda acción es que ésta esté en equilibrio, de tal forma que su tasa requerida de rendimiento sea igual a la tasa esperada de rendimiento: 
$\mathrm{TLR}+\beta\left(\mathrm{R}_{\mathrm{m}}-\mathrm{TLR}\right)=\frac{\mathrm{D}}{\mathrm{P}}+\mathrm{g}$

Tal como es difícil estimar la TLR y PR, es también difícil establecer la g adecuada. Si las tasas de crecimiento y de pago de dividendos se han mantenido estables y si los inversionistas han estado proyectando rentabilidades pasadas (en base al ROE), entonces se puede estimar g como la tasa de crecimiento histórica de la empresa, la cual está dada por:

$$
\mathrm{g}=\operatorname{ROE}(1-\mathrm{d})
$$

Dónde:

Tabla 11. Nomenclatura de la Ecuación 9

\begin{tabular}{lll}
\hline Nomenclatura de la Ecuación 9 & \\
\hline ROE & $d$ & $\begin{array}{l}\text { Rentabilidad sobre el patrimonio } \\
\text { Porcentaje de pago de dividendos a } \\
\text { accionistas }\end{array}$ \\
\hline
\end{tabular}

- Método ROE. - Este es un método interno (puesto que no considera el mercado) y toma en consideración todo el patrimonio, puesto que el ROE (Rotation Over Equity), mide el rendimiento sobre el capital contable común o la tasa de rendimiento sobre la inversión de los accionistas. 


$$
R O E=\frac{\text { Utilidad neta para accionistas }}{\text { Capital Contable Común (patrimonio })}
$$

Recuérdese que, dentro del patrimonio está también la utilidad neta, por lo cual ésta debe extraerse del patrimonio, para no subestimar el índice.

- Tasa Activa más Prima de Riesgo.- Este método aplica para todo el patrimonio y no solamente para las utilidades retenidas. Aunque en realidad es un método subjetivo, los analistas frecuentemente estiman el costo del patrimonio añadiendo una prima de riesgo de 3 a 5 puntos porcentuales a la tasa de interés sobre la deuda a largo plazo de la empresa.

Es lógico suponer que, las empresas que tienen deudas riesgosas, también tendrán un costo de capital riesgoso y de alto costo. Por ejemplo, si una empresa extremadamente fuerte tuviera obligaciones bancarias a largo plazo a una tasa del 18\%, el costo de su capital contable podría estimarse como:

Tabla 12. Cálculo de la tasa requerida por el accionista

\begin{tabular}{ll}
\hline$K_{e}$ & Tasa activa + Prima de Riesgo \\
$K_{e}$ & $18 \%+4 \%$ \\
$K_{e}$ & $22 \%$ \\
\hline
\end{tabular}

Cada vez que la prima de riesgo sea una estimación de juicio, la estimación de Ks será también producto del juicio; por tanto, no es probable que este método produzca un costo preciso para el capital contable. 
Ponderación de los Componentes del Costo.

Cada empresa tiene una estructura óptima de capital, la cual se define como aquella mezcla de deudas y capital contable, que conduce a la maximización del precio de las acciones. Las proporciones óptimas de deudas y capital contable, junto con los costos componentes de capital, se usan para calcular el Costo Promedio Ponderado de Capital.

Por ejemplo, la estructura óptima de capital y costos componentes de una empresa son:

Tabla 13. Estructura óptima de capital

$$
\text { Pasivos }=40 \%
$$

$$
\text { Activos }=100 \%
$$

$$
\text { Patrimonio }=60 \%
$$

Tabla 14. Valores para el cálculo del costo de deuda

\begin{tabular}{ll}
\hline Interés de Deudas: & $16 \%$ \\
Impuestos: & $25 \%$ \\
TLR: & $10 \%$ \\
S\&P 500: & $14 \%$ \\
$\beta:$ & 1.1 \\
\hline
\end{tabular}


Tabla 15. Cálculo del promedio ponderado

\begin{tabular}{|c|c|}
\hline Costo de Deuda & $\mathrm{Kd}(1-\mathrm{T})=16 \%(1-0.25)=12 \%$ \\
\hline Costo de Capital Contable & $\mathrm{TLR}+\beta(\mathrm{Rm}-\mathrm{TLR})=0.1+1.1(0.14-$ \\
\hline $\begin{array}{l}\text { Promedio de los } \\
\text { ponderar) }\end{array}$ & $(12 \%+14.4 \%) / 2=13.2 \%$ \\
\hline Promedio Ponderado de Costos & $12 \%(0.6)+14.4 \%(0.4)=12.92 \%$ \\
\hline
\end{tabular}

Podemos deducir el promedio ponderado de los costos como: el costo de deuda por la proporción de deuda más el costo de capital por la proporción de capital.

La Ecuación del WACC se define como:

$\mathrm{WACC}=\mathrm{K}_{\mathrm{d}}(1-\mathrm{T}) \frac{\mathrm{D}}{(\mathrm{D}+\mathrm{E})}+\mathrm{K}_{\mathrm{e}} \frac{\mathrm{E}}{(\mathrm{D}+\mathrm{E})}$

Usos del WACC

Se puede citar cuatro usos principales del WACC:

- $\quad$ Valoración de nuevas inversiones, proyectos, productos nuevos.- Se usa el WACC a fin de obtener la tasa de descuento a emplearse en los nuevos proyectos. Si la TIR del proyecto es superior al WACC, la empresa estará creando valor para sus accionistas. Si la TIR es inferior al WACC, se estará empobreciendo a los accionistas. 
- $\quad$ A fin de determinar una estructura óptima de financiamiento

- Reordenar los pasivos a largo plazo

- Salidas a Bolsa de Valores.- A fin de Cotizar nuestras acciones en un buen rendimiento

\section{Problemas del WACC}

- Inestabilidad de las tasas de interés en los mercados de capitales.- Las tasas de interés varían mucho; frecuentemente, varían de una semana a otra, pero inclusive pueden variar dentro de la misma semana.

- Problemas de medición.- Como se ha examinado, existen dificultades prácticas al calcular el CAPM, principalmente porque es difícil estimar la prima de riesgo y además, porque no existe la TLR. Por tanto, nunca se podrá estar seguro de cuán exacta será el costo de capital.

- Pesos de la estructura de Capital.- Frecuentemente se dan variaciones en la estructura de capital de la empresa, por motivo de las nuevas deudas y de las nuevas utilidades retenidas. En los ejercicios de este capítulo se ha dado por 
Luis M. Bejar-León; Elaine R. Jijon-Gordillo; Vladimir J. Soria-Freire

sentado la estructura óptima de capital, pero realmente la estructura óptima es una tarea fundamental por sí misma.

Consideraciones para Valuación de Proyecto

- Toda empresa es creada bajo el supuesto de negocio en marcha, es decir, que la empresa es creada para que exista toda una vida y por ende, los flujos de efectivo que se obtengan serán eternos.

- Si no se especifica un crecimiento (g) para los flujos, al momento de obtener el VAN de un proyecto, se debe considerar el último flujo como constante (perpetuidad)

- Si se especifica un crecimiento para los flujos, al momento de obtener el VAN de un proyecto, se deberá considerar el valor continuo de los flujos futuros.

- Si se especifica una duración del proyecto, el VAN será equivalente al valor presente de todos los flujos, sin considerar perpetuidades o valores continuos.

\section{Comparación del Valor Actual Neto Obtenido por los tres Flujos}

Para el análisis se está considerado hasta el momento los tres tipos de flujos citados:

- Flujo de Fondos Libre (Free Cash Flow - FCF), descontado a la tasa WACC

- Flujo de Fondos de Capital (Capital Cash Flow - CCF), descontado a la tasa $\mathrm{WACC}$

- Flujo de Fondos para el inversionista/Accionista (Equity Cash Flow - ECF), descontado al rendimiento del accionista $\mathrm{k}_{\mathrm{e}}$ 
El resultado del VAN del FCF (descontado con la tasa WACC) deberá ser igual al VAN del CCF (descontado con la tasa $\mathrm{WACC}_{\mathrm{BT}}$ ). El VAN obtenido en ambos casos se lo conoce como Valor del Activo Neto.

A diferencia, del resultado de VAN del ECF (descontado a la tasa $\mathrm{K}_{\mathrm{e}}$ ), este valor corresponde al Valor Actual de las Acciones. Para su equivalencia con los dos flujos anteriores, se debe sumar el Valor Actual de la Deuda (se obtiene al descontar los intereses I a su propio costo $\mathrm{K}_{\mathrm{d}}$ ), tendremos como resultado, el Valor del Activo Neto:

$$
\frac{F C F}{W A C C}=\frac{C C F}{W A C C_{b t}}=\left(\frac{E C F}{K_{e}}+\frac{I}{K_{d}}\right)
$$

\section{Método del Valor Presente Ajustado (APV)}

Existe un cuarto método para calcular el Valor del Activo Neto. Este método consiste en descontar el FCF a una tasa que represente la rentabilidad esperada por el accionista, con el supuesto de que la empresa o el proyecto se financian con recursos propios. Esto se refiere, a que el total de activos es igual al total del patrimonio (no existen deudas)

Este método se conoce como Valor Presente Ajustado $^{10}$ (APV), y consiste en obtener un VAN proveniente de descontar el FCF con una tasa "desapalancada" $\mathrm{K}_{\mathrm{a}}$ (el rendimiento del accionista considerando que no existe deuda). Luego se obtiene un VAN producto de descontar el Escudo Fiscal al costo de la deuda $\mathrm{K}_{\mathrm{d}}$. La suma de los dos VAN obtenidos será igual al Valor del Activo Neto.

${ }^{10}$ APV, Adjusted Present Value. Véase Myers, Stewart (1974): "Interactions in Corporate Financing and Investment Decisions - Implications for Capital Budgeting", en Journal of Finance, vol. 29 No. 21. 
Recordemos que el rendimiento se obtiene a través del CAPM y esta tasa esta apalancada debido al coeficiente el beta que incluye financiamiento. Para encontrar la tasa desapalancada $\mathrm{K}_{\mathrm{a}}$ tendremos primero que desapalancar la $\beta_{\mathrm{e}}$ mediante la Ecuación de Hamada ${ }^{11}$ :

$$
\beta_{\mathrm{a}}=\frac{\beta_{\mathrm{e}}}{1+\frac{\mathrm{D}(1-\mathrm{T})}{\mathrm{E}}}
$$

Donde:

Tabla 16. Nomenclatura de ecuación 16

\begin{tabular}{lll}
\hline & $\beta_{a}$ & Beta desapalancada \\
& $\beta_{e}$ & Beta apalancada \\
$\mathrm{D}$ & & Valor de la Deuda \\
$\mathrm{T}$ & $E$ & Tasa Impositiva \\
& & Valor del Patrimonio \\
\hline
\end{tabular}

Con el resultado del coeficiente beta desapalancada $\beta_{\mathrm{a}}$, se procede a obtener la tasa sin deuda $\mathrm{K}_{\mathrm{a}}$ mediante el CAPM:

$$
K_{a}=R_{f}+\beta_{a}\left(R_{m}-R_{f}\right)
$$

Tabla 17. Nomenclatura de la ecuación 17

$\begin{array}{ll}R_{f} & \text { Rendimiento libre de riesgo (Risk Free) } \\ \beta_{a} & \text { Beta desapalancada } \\ R_{m} & \text { Riesgo del mercado }\end{array}$

${ }_{11}$ Véase Hamada, Robert (1972): The Effect of the Firm's Capital Structure on the Systematic Risk of Common Stocks", Journal of Finance, vol. 27 No. 2. 
Por último, se obtiene el VAN del FCF descontado a la tasa $\mathrm{K}_{\mathrm{a}}$. Luego se suma con el VAN del Escudo Fiscal descontado a la tasa $\mathrm{K}_{\mathrm{d}}$ para obtener el Valor del Activo Neto:

$$
\frac{F C F}{K_{a}}+-K_{K_{d}}^{D \cdot T}=\frac{F C F}{K_{a}}+D \cdot T
$$

Donde:

Tabla 18. Nomenclatura de la ecuación 18

\begin{tabular}{lll}
\hline FCF & $K_{a}$ & Flujo de Fondos Libre (FCF) \\
& Tasa de rendimiento (desapalancada) \\
D & $K_{d}$ & Valor de la deuda \\
& Costo de la deuda (tasa de interés) \\
Tasa Impositiva
\end{tabular}

Quedando finalmente equilibrado los resultados del Valor del Activo Neto de la siguiente manera:

$$
\text { ActivoNeto }=\frac{\mathrm{FCF}}{\text { WACC }}=\frac{\mathrm{CCF}}{\mathrm{WACC}_{\mathrm{bt}}}=\left(\frac{\mathrm{ECF}}{\mathrm{K}_{\mathrm{e}}}+\frac{\mathrm{I}}{\mathrm{K}_{\mathrm{d}}}\right)=\left(\frac{\mathrm{FCF}}{\mathrm{K}_{\mathrm{a}}}+\mathrm{D} \cdot \mathrm{T}\right)
$$




\section{Estudio de caso práctico}

The Hampton Machine Company (Caso WACC)

En Febrero de 2003 el Ing. Jorge Andrade, empresario de la República de San Andrés, Sur América, analizaba las informaciones técnicas y financieras sobre un proyecto para incrementar su nivel de producción en The Hampton Machine Company por medio de la compra de una nueva instalación y bodegas, para lo cual deberá invertir hoy $\$ 750,000.00$, obteniendo ingresos durante los siguientes 9 años de $\$ 300,000.00$ anuales y a partir del décimo año se esperan ingresos de $\$ 60,000$ a perpetuidad

Dentro de los puntos más importantes era saber cuál era la tasa de descuento que debería usar para valorar sus flujos y así poder tomar la decisión si debiese dar inicio al proyecto, para lo cual tenía la siguiente información adicional:

- Hampton solicitó un crédito doméstico el 16 de Octubre de 2002 por el valor de $\$ 120,000$ al First Union Bank, el banco no tiene liquidez en ese momento y a cambio le podría dar un Pagaré Bancario a 180 días plazo. Hampton tiene que recurrir a la Bolsa de Valores a negociar el Pagaré y obtener el efectivo; le pide un agente de bolsa le diga cuánto recibiría por la venta del documento y éste le dice \$118,486.01. El Ing. Andrade desea conocer el costo que le estaría cobrando el Banco.

- Compra a crédito de mercadería por \$90,000 el 1ro de Noviembre de 2002 a nuestros proveedores regulares a 91 días.

- Se contrajo un préstamo bancario el 15 de Noviembre del 2001 con The Chase Manhathan Bank (NYC- ROCKEFELLER BUSINESS CENTER) por el valor de $\$ 400,000$ al $11.00 \%$ anual a 2 años plazo (24 meses). Con estos datos calcular los gastos financieros y el saldo de capital del préstamo para el 2002 y compararlos con los datos 
del Estado de Resultados y Balance General. Si el día de hoy el Ing. Andrade decide reestructurar o programar su crédito le cobrarían una tasa del $7.25 \%$ anual

- El 30 de Mayo del 2000, en sesión de directorio la compañía decidió emitir obligaciones a largo plazo por un valor nominal de $\$ 700,000$ (para comprar un lote en el parque industrial de la ciudad), y que vencían el 30 de Mayo del 2005 (base de cálculo comercial). Las características de este documento son las siguientes características:

○ Pago de capital e interés: anual

○ Tasa de interés: pasiva referencial más un margen de dos puntos (TPR inicio cupón vigente era del $4.30 \%$ )

○ Amortización de capital: 10\% primer cupón, 11\% segundo cupón, 12\% tercer cupón, 14\% cuarto cupón y 53\% quinto cupón.

○ Rendimiento nominal: $7.25 \%$

○ Fecha valor 31 de Diciembre 2002

1. Se conoce que Hampton Machine cotiza sus acciones en la bolsa de valores y que el rendimiento mensual en el último año fue de: $18.22 \% ; 24.25 \% ; 18.25 \% ; 20.15 \% ; 21.07 \%$; $21.48 \% ; 22.00 \% ; 22.59 \% ; 19.25 \% ; 22.47 \% ; 22.89 \% ; 25 \%$. Mientras que los rendimientos mensuales del índice de mercado IRECU para ese año fueron del: 11\%; 13\%; $12.45 \% ; 13 \%$; $14 \% ; 12.89 \% ; 13 \% ; 15 \% ; 14 \% ; 16 \% ; 16.50 \% ; 17 \%$. Se estima que la tasa libre de riesgo es del $4.25 \%$. Con estos datos calcule el costo de capital de HAMPTON.

2. El capital social de la corporación está compuesto de 200,000 acciones de valor nominal $\$ 10$ cada acción. En la Bolsa de Valores se cotizan con el \$10.80 la acción. Las utilidades 
retenidas también se toman en consideración para el cálculo del WACC considerando el mismo costo de capital de las acciones.

A continuación se detalla un extracto de los Estados Financieros, Balance General y Resultados, terminados al 31 de diciembre del 2002:

\section{BALANCE GENERAL} Al 31 de Diciembre del 2002

\section{PASIVOS}

Pasivo a corto plazo

Documentos por pagar

Proveedores

Pasivo a largo plazo

Obligaciones bancarias

$194,504.06$

Obligaciones por pagar (emisión)

PATRIMONIO

Capital social

$2,000,000.00$

Utilidades retenidas

$150,000.00$

TOTAL PASIVO Y PATRIMONIO

$3,107,504.06$

\section{ESTADO DE RESULTADOS}

Al 31 de Diciembre del 2002

\begin{tabular}{|lrr|}
\hline VENTAS & $2,400,000.00$ \\
COSTO DE VENTAS & $720,000.00$ \\
UTILIDAD BRUTA & $\mathbf{1 , 6 8 0 , 0 0 0 . 0 0}$ \\
GASTOS GENERALES & $50,000.00$ \\
GASTOS FINANCIEROS & $69,193.79$ \\
$\quad$ Documentos por Pagar & $1,513.99$ \\
$\quad$ Proveedores & 450.00 \\
$\quad$ Obligaciones Bancarias & $32,390.80$ \\
Obligaciones por Pagar (Emisión) & $34,839.00$ \\
\cline { 2 - 3 } UTILIDAD ANTES IMPUESTO & $\mathbf{1 , 5 6 0 , 8 0 6 . 2 1}$ \\
IMPUESTOS Y PTU & $\mathbf{5 6 5 , 7 9 2 . 2 5}$ \\
UTILIDAD DESPUES DE IMPTO Y PTU & $\mathbf{9 9 5 , 0 1 3 . 9 6}$ \\
\hline
\end{tabular}


Ilustración 1. Balance General didáctico

Con los datos expuestos Ing. Andrade recurre a usted que es su staff financiero y le pide que comente o considere sobre si debería invertir la compañía en este momento, para lo cual debe usted realizar un análisis de todos los datos, si están correctas las cifras de los estados financieros y con el cálculo del VPN y TIR de los flujos recomiende si es o no viable el proyecto.

\section{Conclusiones}

En el mundo emprendedor, forjadores de nuevos proyectos de inversión, desconocen la forma adecuada de cómo valorar sus flujos y por ende medir la factibilidad financiera y económica que presentará el proyecto una vez puesto en marcha. El campo de las finanzas en el mundo empresarial es muy relevante, por lo que conocer las herramientas o instrumentos de valoración es fundamental a la hora de brindar información confiable y eficiente a los accionistas y al mercado de capitales, en general.

El Costo Promedio Ponderado de Capital es la mejor alternativa desde el punto de vista que el universo de empresas tienen diversos tipos de financiamiento ajeno y propio, de tal modo que el poder estimar una tasa de descuento apropiada se debe dar en un entorno de suma de los costos de deuda de las principales fuentes de financiamiento externo e interno.

Con base en el estudio realizado en este trabajo y a la experticia en el campo de las Finanzas, soy un testigo del uso y utilización de la tasa promedio ponderado de capital es una herramienta muy útil para la valoración de flujos futuros de proyectos de inversión, debido a que brinda una visualización 
Este método tiene algunos detractores que indican que la tasa promedio ponderado es muy volátil debido a los cambios que existen constantemente en los estados financieros y como consecuencia en la estructura de capital; sin embargo, existen muchas bondades atribuibles a esta herramienta, como la de predecir de manera más real los flujos de fondos con una tasa apropiada que está a la medida del apalancamiento general de los recursos NEC.

\section{Recomendaciones.}

A los interesados en valoración de proyectos de inversión se les recuerda que usar tasas de descuentos provenientes de costos de oportunidad de capital, costo de capital por arbitraje (APT), modelo de fijación de precios de activos de capital, entre otros, en una economía en desarrollo es complicado poder utilizar los métodos mencionados.

Con la finalidad de utilizar el criterio de costo promedio ponderado de capital, podemos estimar el costo de capital a través de algunas suposiciones validas, como por ejemplo, el ROE, tasa de pago de dividendos, costo de oportunidad del inversionista. Con estas tasas podremos eliminar el fantasma de que el método es inviable calcular en una economía que no tiene un mercado de valores desarrollado.

Con el antecedente mencionado, para los interesados en usar este método, siempre que se pueda estimar el costo de deuda de alguna cuenta del pasivo, se deberá hacer una aproximación de costos a fin de tener una tasa más real. Ya que el éxito de esta herramienta es el estimar el costo de deuda de cada una de las cuentas que tienen costo financiero y tener el valor de mercado de los valores que tienen deuda financiera. 


\section{Bibliografía.}

Bernal, C. (2010). Metodología de la investigación. Bogotá: Pearson.

Bonham, A., \& Langdon, K. (2012). Finanzas. Editorial Trillas.

Gitman, L., \& Chad, Z. (2012). Principios de Administración Financiera. México: Pearson.

Hamada, R. (1972). The Effect of the Firm's Capital Structure on the Systematic Risk of Common Stocks. Journal of Finance, Vol. 27 No. 2.

Hernández Sampieri, R., Fernández Collado, C., \& Baptista Lucio, P. (2010). Metodología de la Investigación. México: McGraw-Hill Interoamericana.

Miles, James, \& Ezzel, J. (Septiembre de 1980). The Weighted Average Cost of Capital, Perfect Capital Markets and Project Life: A Clarification. Journal of Financial and Quantitative Analysis, 719 - 730.

Myers, S. (1974). APV, Adjusted Present Value. Interactions in Corporate Financing and Investment Decisions - Implications for Capital Budgeting. Journal of Finance, Vol. 29 No. 21.

Ochoa Setzer, G. A., \& Saldívar del Ángel, R. (2012). Administración financiera correlacionada con las NIIF. México: Editorial McGrawHill.

Ross, S. A., Westerfield, R. W., \& Jaffe, J. F. (2012). Finanzas Corporativa (Novena ed.). México: Editorial McGrawHill.

Sapag Chain, N. (2011). Proyecto de inversión, formulación y evaluación. Macul: Pearson.

Sharpe, W. (Septiembre de 1964). CAPM, Capital Asset Pricing Model. Capital Asset Prices: A Theory of Market Equilibrium Ander Condition of Risk. Journal of Finance, 277 - 293. 